\title{
Household demand for sanitation improvements in low-income informal settlements: A case of East African cities
}

\author{
K. Okurut ${ }^{1,3}$ and K. J. Charles ${ }^{2}$ \\ ${ }^{1}$ Centre for Environmental Strategy (CES), University of Surrey, Guildford, UK \\ ${ }^{2}$ School of Geography and the Environment, University of Oxford, OX1 $3 Q Y, U K$ \\ ${ }^{3}$ Departments of Civil and Environmental Engineering-CEDAT, Makerere University, Kampala, \\ Uganda
}

\begin{abstract}
Informal urban settlements present a range of challenges to sanitation provision, including low incomes, insecure of tenure, low education levels, difficult topography and transitory populations. A household stratified probability survey complemented with focus group discussions and interviews was undertaken in low-income informal settlements in Kigali, Rwanda; Kampala, Uganda; and Kisumu, Kenya, to assess the household sanitation demand status and identify the barriers and catalysts to demand for sanitation improvements in these areas and between the cities.

A five progressive decision-stage sanitation demand model revealed that similar proportions of respondents had already installed systems in Kigali (13.2\%) and Kampala (12.5\%). However, there was a higher proportion in Kigali for each of the categories of Preference, Intent and Choice. In Kisumu, only 3.2\% of respondents indicated that they had considered installing (or installed) a household sanitation facility. Reported barriers and catalysts varied between the demand stages and across the cities.

The differences in attitudes at the stages of demand, and between these three cities, highlight the need to tailor programmes to meet the local demand for sanitation improvements, specific for each community.
\end{abstract}

Key words: Barrier, Demand, East Africa, Improved sanitation, Informal settlements.

\section{Introduction}

The challenges of providing safe and adequate sanitation in informal settlements for improved health and sustainable livelihood are known to include social, environmental, economic, institutional and demographic characteristics (Isunju et al. 2011). Low-income informal settlements are commonly characterized by transient urban poor locals who cannot easily afford basic services and present unique challenges to urban authorities (UNHABITAT 2003; Foppen and Kansiime 2009). Many dwellers may continue to live in these conditions because they are "trapped in poverty" and cannot move on to the formal urban lifestyle in the competitive market forces offered by the city (Marx et al. 2013).

Due to the unique challenges in the provision of services in informal settlements, basic sanitation coverage is much lower compared to the average for urban areas (Foppen and Kansiime 2009). Where facilities exist, they are likely to be shared, unhygienic, and not afford the users dignity and privacy (Van Der Geest 2002; Tumwebaze et al. 2013). 
Efforts to improve access to sanitation have mainly focused on hardware interventions (Murray and Ray 2010; Van der Hoek et al. 2010). This can be linked to the general understanding of progress as reported by the Joint Monitoring Programme (JMP) on sanitation which considers the number of facilities installed (WHO/UNICEF 2010). Hardware intervention may be necessary as direct support to the economically most vulnerable households/communities who may not afford adequate sanitation from a human rights perspective. Provision of sanitation hardware without considering the local demand may result in the facilities either being abandoned/misused or never used at all (Solo et al. 1993; Mara et al. 2010) which amounts to a wastage of resources. Integrated approaches identify the software attributes necessary for any sanitation hardware interventions, through the involvement of all stakeholders (Van der Hoek et al. 2010; Lüthi et al. 2011; Mosler 2012) and has proved useful in identifying strategies for scaling-up sustainable access to improved sanitation (Varley et al. 1996; Robbins 2007) in low-income informal settlements.

Adequate sanitation should observe the principles and practices of collection, removal or storage and disposal or re-use of human excreta with the concept of privacy and dignity from a human rights perspective as recognised by the United Nations bodies (COHRE et al. 2008). The realisation of full public health and socio-economic benefits of good sanitation will not only depend on the presence of sanitation hardware, but also, on its indiscriminate and continuous usage (Schertenleib 2001; Mara et al. 2010; Van der Hoek et al. 2010). Based on this argument, this article adopts the definition of sanitation developed by the Millennium Task Force as "access to, and use of excreta and wastewater facilities and services that ensure privacy and dignity, ensuring a clean and healthy living environment for all" (COHRE et al. 2008).

Improved sanitation technology that hygienically separates human excreta from human contact (WHO/UNICEF 2010), and meets the conditions of safety, accessibility, acceptability, privacy, dignity and cleanliness as required from the human rights points of view; qualifies to be an improved sanitation facility. Sanitation technologies that are expected to meet the conditions include: flush/pour flush toilets connected to a sewer system, a septic tank, or a pit latrine; ventilated improved pit (VIP) latrine; pit latrine with a slab; composting toilet and other special cases (WHO/UNICEF 2010). However, the system may not provide full public health and socio-economic benefits to the household/community if it does not give access, privacy, dignity, cleanliness, and is affordable.

Many reasons have been put across to explain the slow progress in achieving the Millennium Development Goal Target 7(c): to halve the proportion of people without sustainable access to safe drinking water and basic sanitation by 2015 (UN 2002), and include demographic, technological, financial, regulatory, institutional, and political reasons (Isunju et al. 2011; Kariuki 2011) but the one aspect that still comes out clearly is the conventional supply-led model that has failed to generate demand for improved sanitation among targeted households (WSP 2001; Jenkins and Sugden 2006; Roma et al. 2010). Lessons learnt worldwide show that the provision of facilities does not guarantee proper usage, and efforts should not only reduce the huge number who remain without access to a toilet but also the huge number who do not use facilities hygienically even when they are available (Peal et al. 2010; Tumwebaze 
et al. 2013). Understanding the local demand will guide in identifying appropriate software attributes to go with the hardware so as to increase acceptability and usage. The attributes may involve: empower users with knowledge, enable a change in behaviour, create demand for services, facilitate establishment of supply chains, improve the planning and implementation of hygiene and sanitation projects to go with appropriate hardware (Evans 2004; Van Wyk et al. 2004; Peal et al. 2010). Hence, the importance to understand the households demand status and identify the barriers and catalysts at the different stages in the sanitation demand decision process model, in order to develop appropriate intervention strategies (Jenkins and Scott 2007).

The demand for improved sanitation services is not a simple concept as it is influenced by a number of factors that include among others: demographic characteristics, availability, reliability, cost and convenience, household situations and attitudes, household awareness and understanding, technical, competing priorities, tenancy and geophysical settings (ParryJones 1999; Jenkins and Sugden 2006).

Drawing from the works of Jenkins and Scott (2007) and Parry-Jones (1999), we consider household demand for sanitation improvement as a decision behavioural process based on psychological, economic and engineering theories and defined as a process of an informed expression of willingness, and ability, to adapt to a new or better sanitation and appropriate sanitation services of preference (Okurut et al. 2014a). Behaviour change can create demand for sanitation improvement, and numerous models have been suggested to assess behaviour change (Devine 2009; Mosler 2012; Dreibelbis et al. 2013) and demand (Jenkins and Scott 2007; Santos et al. 2011a) for sanitation improvements. This study has adapted the three progressive decision stages model developed by Jenkins and Scott (2007) and, extended to a five stage model to include those who have not considered installing an improved sanitation system (No preference) and the actualized category of installed (Figure 1).

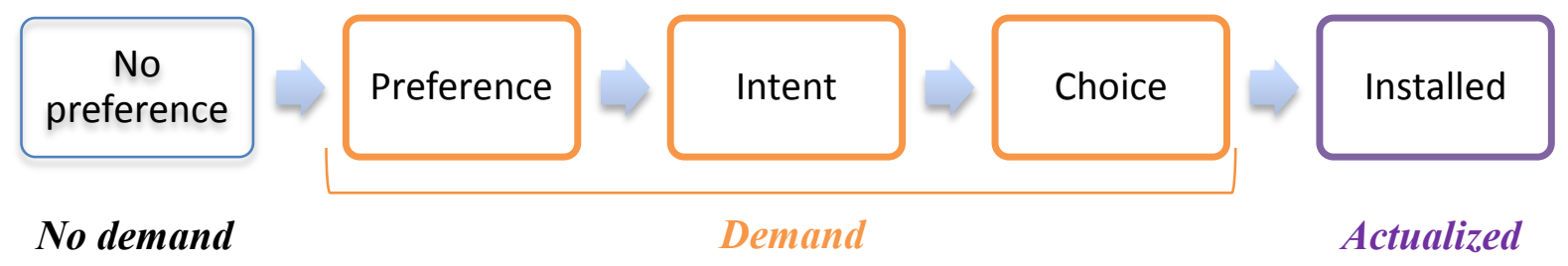

Figure 1: Household demand model adopted from (Jenkins and Scott 2007)

In the model "Preference" indicates that the household has considered installing a household sanitation facility (toilet), but do not intend to install within a year; "Intent" indicates they have a low to medium likelihood of installing within a year; and "Choice" indicates a high likelihood of installing within a year and "Installed" is respondents who have indicated that they have already installed a household sanitation facility. Preference, intent and choice are further grouped into "Demand".

This paper aimed to characterize demand for household sanitation improvements and builds on household survey findings on sanitation study that identified barriers and catalysts to improved sanitation in informal settlements in East Africa (Charles and Okurut 2013). The study involving a multi-disciplinary team has used mixed methods to assess the household 
sanitation demand characteristics and point out barriers and motivations to demand for improvements. Any intervention in the settlements would then be designed specifically to address the barriers and facilitate the motivations to stimulate demand for improved sustainable sanitation services at households. Where there is an informed and expressed demand for sanitation improvements, interventions designed to meet the local demand will highly be acceptable and adopted by the target households (Evans 2004; Mara et al. 2010; Peal et al. 2010).

\section{Methodology}

A diagnostic study of the sanitation situation in three case study cities: Kampala (Uganda); Kigali (Rwanda); and Kisumu (Kenya) was conducted to understand what is known about the cities. The diagnostic report aided a purposive selection of eight low-income informal settlements for the study. The study settlements selected have urban characteristics with high population densities and poor neighbourhoods, and have been reported to have sanitation related problems. The study used household stratified probability survey in the selected lowincome informal settlements as the main research method and supplemented with transect walks, focus group discussions (FGDs) and interviews.

The study sample size in each city was determined using a simplified sample size formula for proportions (Israel 1992). The survey questionnaires were administered to heads or adult members of household samples selected through random route sampling techniques in proportion to the population of study area. The samples sizes for Kigali $(n=1794)$, Kampala $(n=1666)$ and Kisumu $(n=1927)$ were determined using the national statistics of the study settlements for the three cities, and were large enough to allow for comparative analysis of sub-groups of households of different socio-economic status (NISR 2008; KNBS 2010; UBOS 2011). Boundaries of the primary sample units (cells / zones / villages) in each study settlement were first identified with the help of the respective local authorities (guides) during transect walks through the settlements. The researchers then randomly selected a number of routes with clearly identifiable physical features through the primary sample unit and, by walking along every route from the start to end; the $\mathrm{n}^{\text {th }}$ household was systematically selected to constitute the study sample until the required households for each route is completed. To ensure that every household in the settlement had an equal chance of being selected, 10 households were systematically selected along each random route and the number of random routes in each primary sample unit was determined as a proportion of the total sample required for the whole settlement.

Findings from the household survey were used to develop qualitative tools for more in-depth understanding (Johnson and Onwuegbuzie 2004) of access to improved sanitation from the perspectives of key stakeholders in the sector. Samples for the FGDs were purposively selected with a target of having the most productive sample to answer the questions with a size that adequately answers the research questions until new explanations stop emerging from the data (Marshall 1996). Interviews were conducted when appropriate sized sample for a group (6 to 12 persons) could not be found for the particular group. The study conducted 83 FGDs by gender, except for local authorities, and 99 interviews: 23 focus group discussions 
and 28 interviews in Kampala, 26 focus groups and 28 interviews in Kigali and 34 focus group and 43 interviews in Kisumu between March and July 2013. Interview and FGD guides were developed for each city using the findings of the household surveys. The researcher facilitated the FGDs and conducted each interview in a language best understood by the group or individual, and later translated the responses into English. The groups or individuals were encouraged to express their views, and interesting points were followed-up with prompts and probes, to get more in-depth understanding of demand for sanitation improvements. The survey questionnaire, FGD and interview guides were pilot tested before being administered in the communities, and all the staff involved with the data collection were familiar with the local languages and were trained before conducting the field work. The methodology was given a favourable response from the Ethics Committee at the University of Surrey.

Due to the difference in the cost of living in the three countries and the inaccurate income data, deprivation was used as a multidimensional scale to measure the poverty levels across the cities. Variables on ability to afford basic needs were used to construct an index that could compare with the level of deprivation of a household across the scale and then normalised to have distributions around the mean for the study samples and for each country.

The Statistical Package for the Social Sciences (SPSS version 20) was used to analyse the household survey data for frequencies, rates and proportions. Cross tabulations were carried out to examine relationships between variables. Pearson Chi-Square values were used to determine the strength of relationship between variables at a $95 \%$ significance level.

Households in the category of "installed" were further broken down as "installed and improved systems" and "installed but unimproved systems". The reason for category splitting is because not all the installed facilities were improved as to provide safety, access, privacy, dignity and cleanliness for full public health and socio-economic benefits and thus considering such respondents to have improved systems is not right. In this paper, "installed and improved" system implies the household has improved technology with conditions that provide safety, access, privacy, dignity and cleanliness likely to result in full public health and socio-economic benefits. While for "installed but unimproved" systems, the household may have either installed an unimproved technology or installed an improved technology but the conditions of the facility does not provide adequate safety, access, privacy, dignity and cleanliness for full public health and socio-economic benefits (COHRE et al. 2008). An "installed but unimproved" system may have a negative impact in the neighbourhood environment of the community. In this case, the respondent may need education to consider improving their system or installing a new better system.

\section{Results and discussions}

\section{Demographics}

The cities had different demographic characteristics in their low-income informal settlements: Kigali had the lowest levels of tenancy and lowest levels of deprivation. Kampala had the 
highest levels of education, but a moderate level of deprivation. Kisumu had high rates of tenancy Figure 2.

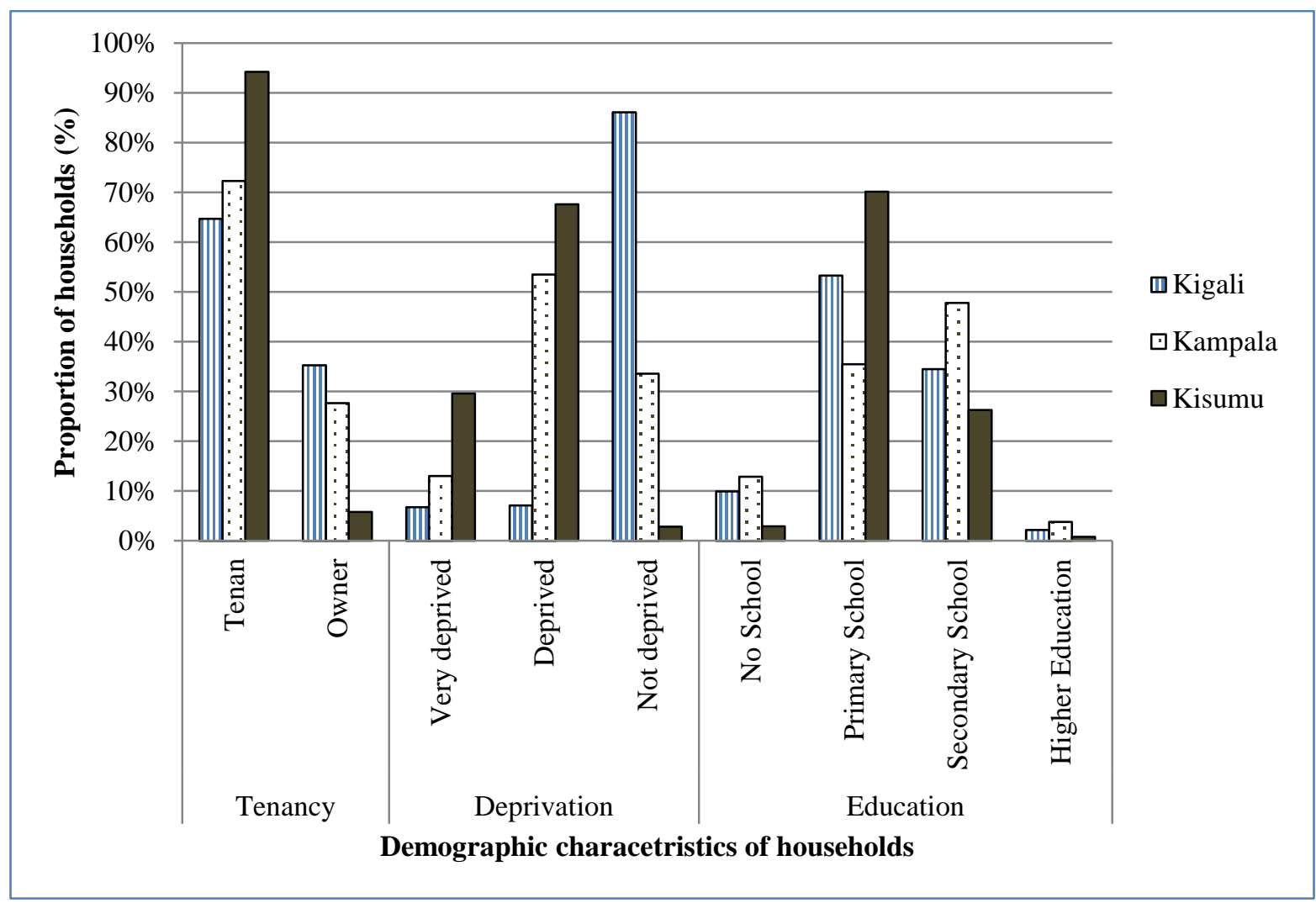

Figure 2: Variation of demographic characteristics between study cities

Majority of the respondents were educated up to primary/secondary level, the highest in Kisumu though Kampala reported the highest proportion of respondents with higher education level.

The difference in demographic characteristic of households in the settlements and between cities highlights the fact that the households may be at different stages of considering to invest in sanitation facilities. Property ownership can influence the likelihood of a household to invest in infrastructural developments. Compared to owner-occupiers, tenants do not own the property they live in, and pay high rent premiums to live close proximity to the city which reduce their opportunities to accumulate savings for investments (Günther et al. 2011; Marx et al. 2013).

\section{Sanitation facilities}

A majority of the respondents self-reported to have some form of sanitation facility with only a small percentage $(6.3 \%)$ indicating that they practice open defecation. According to the JMP classification, more than half $(59.7 \%)$ of the type of sanitation facilities reported by the respondents fall in the category of improved sanitation technologies (Kisumu $65.4 \%$, Kampala, $56.9 \%$ and Kigali $56.2 \%$ ). However, subjecting the facilities to the problems reported by the respondents, a high proportion of these facilities did not provide "sustainable access to basic sanitation" based on safety, privacy, dignity, cleanliness and healthy 
environment for all. Over $95 \%$ of the facilities were regarded not to provide full public health and socio-economic benefit to the users (Okurut et al. 2013).

\section{Sanitation demand}

The household survey revealed that less than $12 \%$ of the households have considered installing a household improved sanitation facility (install new or upgrade existing facility) with interesting differences between the cities (Figure 3).

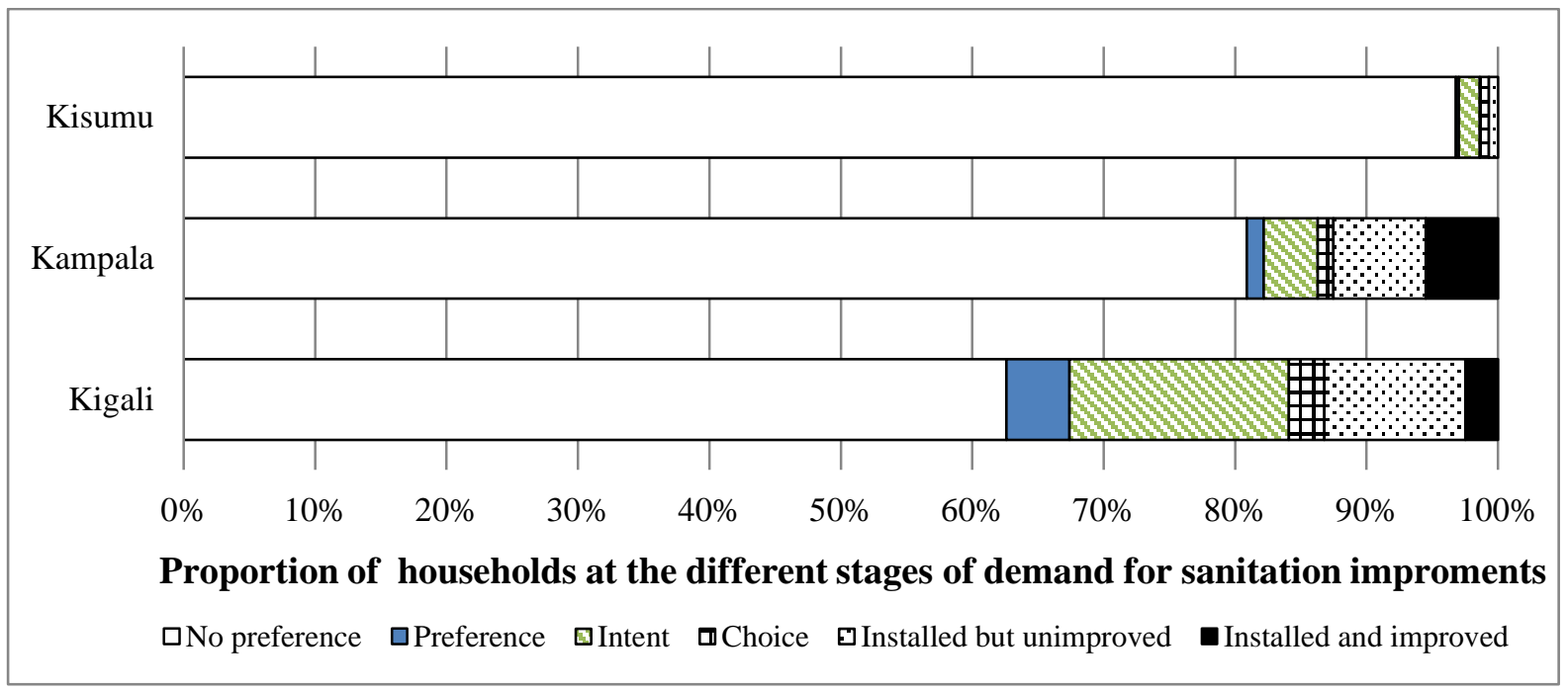

Figure 3: Household sanitation demand status (significantly different between the cities $p<0.0005$ )

While similar proportions of respondents have already installed systems in Kigali (13.2\%) and Kampala (12.5\%), there was a higher proportion in Kigali for each of the categories of preference, intent and choice. In Kisumu, less than $1.0 \%$ of respondents have installed a household sanitation system. These proportions relate with the demographic characteristics of the settlements in the three cities and support the argument that tenants are less likely to invest in infrastructural improvements within informal settlements (Marx et al. 2013). Of the respondents with no improved systems to provide full public health and socio-economic benefits, less than 5\% where likely to install within a period of 12 months: Kampala (1.7\%), Kigali $(3.1 \%)$ and Kisumu $(0.7 \%)$. Few studies have assessed demand for sanitation improvements based on the "preference", "intent" and "choice" models (Okurut et al. 2014b). However, a sanitation demand study found to be more comparable was conducted in Ghana, where a low rate of new demand (5.8\%) was also reported among households without improved systems (Jenkins and Scott 2007). Comparison of the two studies in peri-urban and low-income informal settlements implies that demand for household sanitation improvement is associated with the type of settlements and household income level.

Though the proportion of households that have actualized (adopters) in Figure 3 can be comparable to the study in Ghana were $11 \%$ was reported, the authors did not consider the fact that some households could have reported to have installed when the conditions of the facilities may be inadequate (Jenkins and Scott 2007). Subjecting the already installed facilities reported in this study to conditions required from the human rights perspective suggests only $2.5 \%$ of the households with actualized facilities have adequate facilities. 
About $6 \%$ of the respondents who indicated to already have installed will require some interventions to help them upgrade to adequate sanitation to meet the human right to sanitation, and to realize full public health and socio-economic benefits. Residents expressed similar views in focus group discussions, that most of the existing facilities in the settlements do not provide full public health benefits. In a study in Darkhan, Mongolia; the authors also report that it is possible to have facilities in peri-urban areas largely regarded as improved by the JMP definition, but some actions are still needed to improve the conditions to provide full public health to the users (Sigel et al. 2012). In another study in Kampala, Uganda; the authors report high access to sanitation facilities in slums, but most of them were shared and majority of the respondents were not satisfied with their facilities, primarily due to cleanliness and high number of users per stance (Tumwebaze et al. 2013; Tumwebaze et al. 2014). The identification in this study of an additional category of households with inadequate improved sanitation technologies that should not be ignored, comes from the strength of using mixed methods to look beyond the response that "have already installed". The knowledge that there may be a category of households not considering to install or upgrade, with a wrong view of what is improved is important in identifying appropriate interventions for their situation. Identification of barriers and development of appropriate strategies can improve access to adequate sanitation in low-income informal settlements.

\section{Demand barriers}

The study shows that "lack of space for a toilet" (42.0\%) or "inability to afford to install a toilet" $(39.3 \%)$ were the main reasons reported for lacking a sanitation facility among the open defecators in Kisumu (17.3\%), which is comparable with findings of a study in Ghana that reported space (48.4\%) and high cost $(33.6 \%)$ as the constraints to constructing toilets (Jenkins and Scott 2007).

"If more space can be provided and access roads provided then I can upgrade the facilities since they can be emptied by a mechanized emptier. If sewerage lines can be extended to our area and connectivity made cheaper, I can upgrade to water borne systems for a few of the tenants that would afford to pay", Landlord, Kisumu, Interview.

Among the house owners (owner occupiers and resident landlords) in the study cities, lack of money (cannot afford) came out as the main barrier faced in building household sanitation (56.2\%), followed by topography (28.4\%) and then lack of space (11.6\%). In Kampala, topography which includes: high ground water tables, collapsing soils, and rocky grounds; was reported as the biggest barrier faced in building sanitation facilities (60.5\%). Lack of money was reported as the main barriers in both Kisumu (75.8\%) and Kigali (68.2\%).

The variation in barriers to demand for sanitation improvement relate to the local geophysical and demographic characteristics of the settlements (Okurut et al. 2013) and will thus require specific intervention for each locality.

\section{Barriers at the different demand stages}


Comparing each of the barriers to building a sanitation facility along the demand decision stages show significant $(\mathrm{p}<0.0005)$ variation on how the barriers are perceived by households at "preference", "intent" and "choice" (Figure 4).

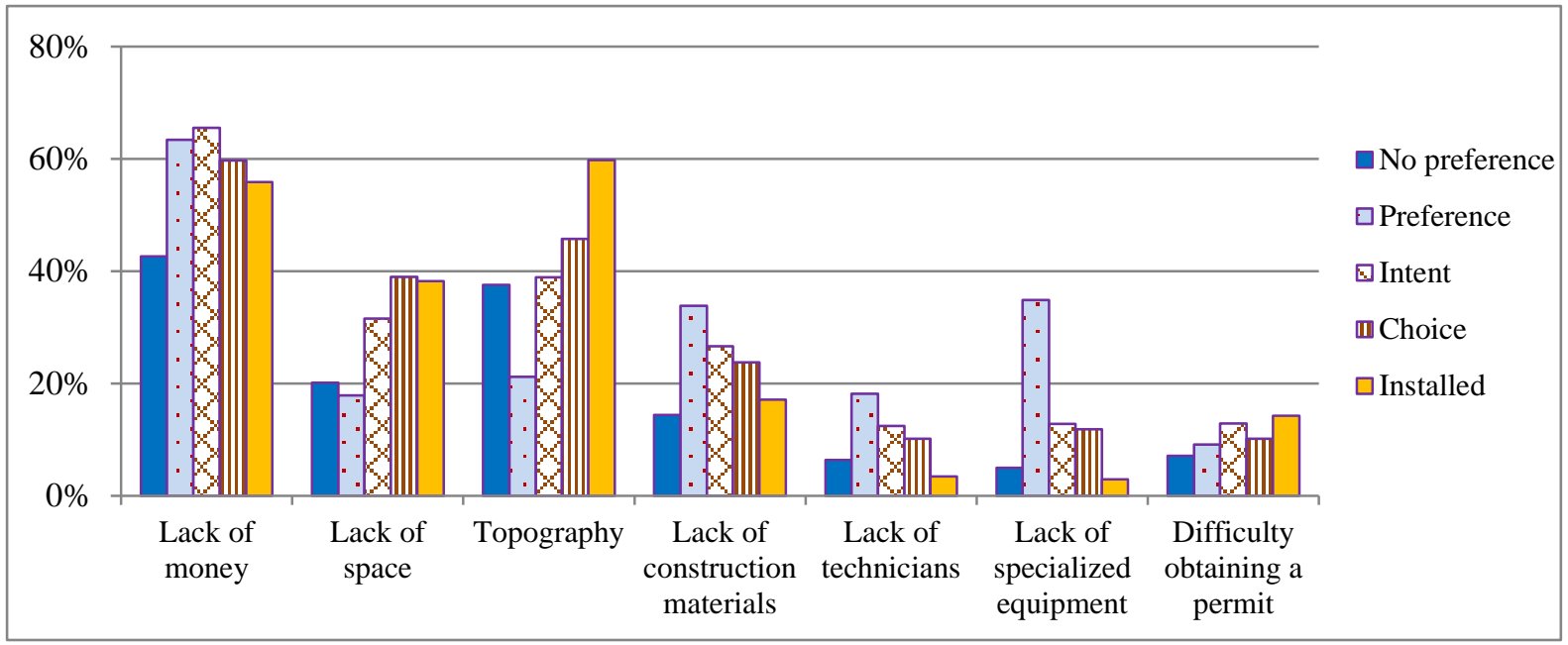

Figure 4: Barriers to building private sanitation facility, reported by own occupiers

Generally, many of the factors decreased in importance from preference to intent to choice to install. Those that increased included: lack of space, topography, and difficulty obtaining a permit. Difficulty in obtaining a permit increased along this chain, indicating that perhaps this is not something that people were aware of at the start of the process. Culture and beliefs, and lack of information were small concerns (less than 1\%). Using the Contingent Valuation method in Ghana, Whittington et al. (1993) also found that social or cultural variables had little effect on household demand for improved sanitation.

However, from FGDs and interviews with key informants, culture was perceived as a big issue in informal settlements. Participants argued that because the communities are inhabited by people from different backgrounds, the sanitation practices are related to the varied cultures. An example given was that some cultures prohibit pregnant women from defecating in a toilet because of the belief that the foetus may fall into the pit, and they are encouraged to use the bush.

Even with the barriers, to find that some households were already at different stages of the demand decision process implies that there should be other factors that encourage them to consider improving their sanitation systems. Identification of motivation factors to demand for sanitation improvement is necessary to know what will encourage the households despite other hindrances, to demand for improved sanitation.

\section{Demand catalysts}

With regard to the factors that motivate demand for sanitation improvements, the study identified the relative importance of different factors in influencing the type of sanitation a household prefers. Prestige/status, culture, law, health and hygiene, privacy, safety, cleanliness, comfort and cost were the factors that were weighed in four levels of importance as: very important, important, less important and not import; in influencing the type of sanitation one would prefer. The results show difference in the weight of importance between 
cities and at the different stages of demand for sanitation improvements. In general, the three very important motivation factors included cleanliness $(71.1 \%)$, health and hygiene $(69.9 \%)$ and privacy (63.5\%), with some respondents urging in the FGDs that their local culture compels them to maintain good toilets. Other scholars have also reported good health and cleanliness as some of the most important factors that motivate households to install improved sanitations (Jenkins and Scott 2007; Isunju et al. 2011; Santos et al. 2011b). A similar view was also echoed during focus group discussions with residents.

"Cleanliness should be priority because it has other benefits beyond money: "You cannot leave your child and toilet dirty because you are working too hard, after all this child will get sick and all your hard earned money will go to treating your child”, Female youth, Kigali, FGD.

By city, more respondents in Kigali and Kisumu indicated importance on health and hygiene and cleanliness than in Kampala. There were many who were concerned with law in Kampala compared to Kigali and Kisumu.

From the focus group discussion and interviews, a number of other factors were recognised to motivate landlords to provide their tenants with improved sanitation.

"Once you have a good toilet, you can even increase the prices for the rent and even some tenants will not have any problem agreeing to increase the rent. However, the issue is to get the money and upgrade the house and at the same time improve the toilet", Landlord, Kigali, FGD.

"I can also upgrade if the whole community is also doing the same so that we all have a clean environment", Landlord, Kisumu, Interview.

The study has shown that some factors may be barriers in one settlement or at some stage of demand, but may also act as a catalyst in another settlement or stage of demand. It implies that any factor that can motivate a household to demand for improvements should be facilitated to encourage adoption to better sanitation as each factor may play a big role to progress a household from one stage to the next stage of demand. This is supported by other studies on culture for instance, in Kenya, the authors reported that some communities believe in defecating in the open because of their cultural influences (WSP 2004), while another study in Uganda reported high sanitation coverage in south-western Uganda largely because of cultural beliefs which condemn households without latrine facilities (Outlaw et al. 2007). Where any factor can have a positive impact in stimulating demand to improve access to adequate sanitation in an area, efforts should be put to promote it in the community.

\section{Demand and household socio-economic status}

Comparison of the level of demand for improved sanitation facility among owner occupiers and tenants along the demand stages show significant $(p<0.0005)$ variation between the cities with owners more likely to have installed a system in Kampala than in the other cities while Kisumu had the highest proportion of tenants with no preference. Generally, there were a higher proportion of owners in the preference, intent and choice stages than tenants across all cities (Figure 5). 


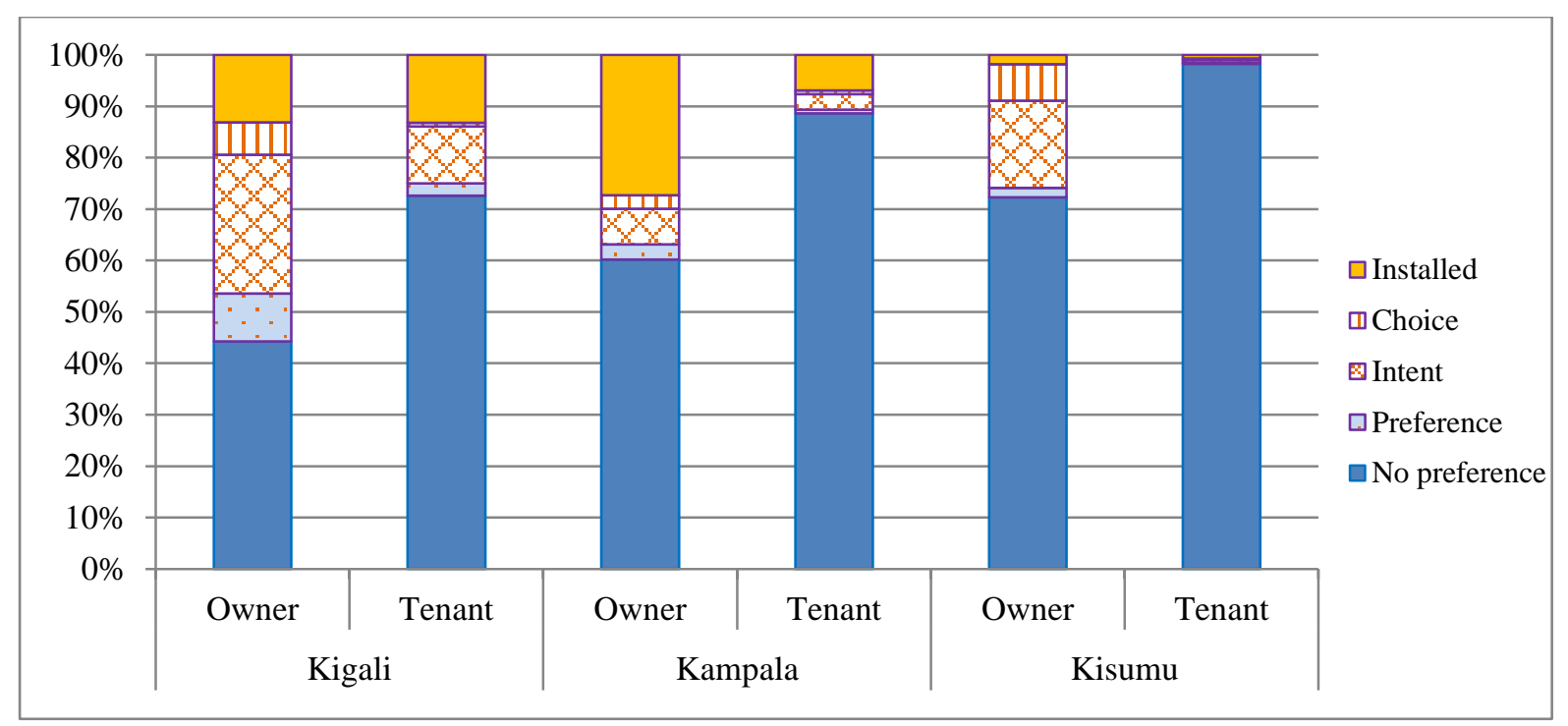

Figure 5: Own occupiers versus tenants along the demand stages

The study shows that tenancy status has some influences on the household demand for sanitation improvement and likelihood of tenant/own occupiers to have installed a toilet varies between the cities.

"Majority of the people here are tenants; we keep shifting and therefore do not care to keep good sanitation. Poverty is also a cause as people work too hard and barely have enough time to clean up their children and their houses" Female youth, Kigali, FGD.

While tenancy was a bigger barrier in Kampala where $60.3 \%$ of those who had installed owned their property compared to only $35.2 \%$ in Kigali. Tenancy issues have also been noted to influence demand for sanitation improvements in other studies (Jenkins and Sugden 2006; Jenkins and Scott 2007).

"Tenants cannot ask for improvements because before a tenant enters the house; I inform them in advance that there is no toilet facility. In this way they agree knowing what is available and will endure the situation", Male landlords, Kampala, FGD.

With regard to education, there were more respondents with higher education $(25.6 \%)$ who have installed a sanitation facility compared to no schooling $(11.5 \%)$, primary school $(7.0 \%)$ and secondary school $(8.8 \%)$. Though more than half $(58.1 \%)$ of respondents with higher education reported no preference in sanitation demand status, it was still lower compared to those with no education (76.6\%), primary school (84.0\%) and secondary school $(77.5 \%)$. The increased demand for sanitation improvement with higher levels of education was also reported in Kenya where households with members who had a higher level of literacy were most likely to demand and adopt safer methods of excreta disposal than those with low levels of literacy (WSP 2004).

Comparison of demand and deprivation of the respondents show that those who are "not deprived" are more likely to have a sanitation facility installed (16.2\%) than the "deprived" and still a majority of those who were "not deprived" have "considered installing" a private sanitation facility. Most of the respondents who indicated no preference were deprived and those in the intent and choice stages significantly varied across the cities. The majority of the 
very deprived respondents who indicated to have considered installing a sanitation system in Kigali were at the intent stage which happens to be higher than in the other cities too. The results also show that it is more likely for the "not deprived" households to have a sanitation facility "installed" in Kampala and Kisumu than either the deprived or very deprived. While in Kigali, households that have a sanitation facility "installed" were mostly deprived.

\section{Conclusion and recommendation}

Though more than half (59.7\%) of the type of sanitation facilities reported by the respondents fall in the category of improved sanitation technologies, a high proportion of these facilities did not provide "sustainable access to basic sanitation" based on privacy, dignity, cleanliness and healthy environment for all. The majority of facilities either shared with one or more households do not afford privacy to the users or were not available when needed by the households (either locked at night or have long queues during the day), and thus did not provide the basic conditions required for improved sanitation facilities (COHRE et al. 2008).

Less than $12 \%$ of the households without improved sanitation facilities have considered installing a household improved sanitation facility with less than $5 \%$ considering installing in the next 12 months. Also, some households with unimproved facilities were reluctant to install better systems as they thought what they had was adequate for good health. Such households may require specific programmes designed to help them upgrade and realise the full public health and socio-economic benefits of improved sanitation facilities. The low level of demand for sanitation improvements in low-income informal settlements comes from the lack of space to install a sanitation facility, inability to afford to install or upgrade to an improved sanitation facility, topography of the settlements, and property rights where majority of the inhabitants are rent-paying tenants. However, it is important to note the household perception on barriers change as the household progresses on the demand stages. Cleanliness, health and hygiene, and privacy are the key factors that motivate households to demand for sanitation improvements. Landlords are also motivated by law, increased earnings from high rental fees, and whether other people are also improving their sanitation facilities in the neighbourhood.

The differences in attitudes between the three cities studied, and between the stages of demand, highlight the need to tailor programmes to meet demand for sanitation improvement conditioned to the communities' needs. Where any factor can have a positive impact in stimulating demand to improve access to adequate sanitation in an area, efforts should be put in to promote it in the community. Interventions in low-income informal settlements should compel landlords to provide housing services with improved sanitation facilities, and subsidies or incentives should be targeted to benefit the most vulnerable. Appropriate interventions should be identified for the category of households at each stage of demand to facilitate their progression to actualization. There is also need for a more open and well regulated market that provides alternative technologies appropriate for the geographical context. 


\section{Acknowledgements}

This study forms part of the $3 \mathrm{~K}-\mathrm{SAN}$ project that is funded by European Union under the SPLASH Sanitation programme. The authors would like to thank: P. Abbott, J. Chenoweth, T. Kaime, R. Kulabako, R. Malcolm, L. Okotto and S. Pedley for the study design; A. Tsinda and J.M. Adogo for the study design and data collection; and the research assistants who were involved in the collection and entry of data in the three cities; Y. Karuhanga, L. Ninsiima, C. Tuyishime, G. Ngoboka, E. Munyeman, D. Onyango and M. Wayumba.

\section{Reference}

Charles, K. and K. Okurut. 2013. Identifying barrier and catalysts to improved sanitation in informal settlements in East Africa: Poster presentation. Water and Health Conference: Where Science Meets Policy. University of North Carolina-ChapelHill, USA: UNC.

COHRE, WaterAid, SDC, and UN-HABITAT. 2008. Sanitation: A human rights imperative. pp. 50. Geneva.

Devine, J. 2009. Introducing SaniFOAM: a framework to analyze sanitation behaviors to design effective sanitation programs. Learning to scale up. Working paper). Washington, DC: WSP.

Dreibelbis, R., P. J. Winch, E. Leontsini, K. R. Hulland, P. K. Ram, L. Unicomb, and S. P. Luby. 2013. The integrated behavioural model for water, sanitation, and hygiene: a systematic review of behavioural models and a framework for designing and evaluating behaviour change interventions in infrastructure-restricted settings. BMC public health 13(1): 1015.

Evans, B. 2004. Whatever Happened to Sanitation?-Practical steps to achieving a core Development Goal. MDG Task Force background paper. New York.

Foppen, J. W. and F. Kansiime. 2009. SCUSA: integrated approaches and strategies to address the sanitation crisis in unsewered slum areas in African mega-cities. Reviews in Environmental Science and Biotechnology 8(4): 305-11.

Günther, I., A. Horst, C. Lüthi, H.-J. Mosler, B. C. Niwagaba, and I. Tumwebaze K. 2011. Research evidence for policy. Where do Kampala's poor "go"? Urban sanitation conditions in Kampala's low-income areas. pp. 4. Kampala: Makerere University.

Israel, G. D. 1992. Determining sample size: University of Florida Cooperative Extension Service, Institute of Food and Agriculture Sciences, EDIS.

Isunju, J., K. Schwartz, M. Schouten, W. Johnson, and M. Van Dijk. 2011. Socio-economic aspects of improved sanitation in slums: A review. Public Health 125: 368-76.

Jenkins, M. W. and B. Scott. 2007. Behavioral indicators of household decision-making and demand for sanitation and potential gains from social marketing in Ghana. Social Science \& Medicine 64(12): 2427-42.

Jenkins, M. W. and S. Sugden. 2006. Rethinking sanitation: Lessons and innovation for sustainability and success in the new millennium. Human Development Occasional Papers (1992-2007). New York.

Johnson, R. B. and A. J. Onwuegbuzie. 2004. Mixed methods research: A research paradigm whose time has come. Educational Researcher 33(7): 14-26.

Kariuki, P. 2011. The challenges of financing sanitation in sub-saharan countries African: Acritical perspective. Journal of Applied Technology in Environmental Sanitation 1(1): 9-16.

KNBS. 2010. Population and Housing Census 2009 A, B, C and VII. K. N. B. o. S. (KNBS). Nairobi: Government printers.

Lüthi, C., A. Morel, E. Tilley, and L. Ulrich. 2011. Community-Led Urban Environmental Sanitation Planning (CLUES). Swiss Federal Institute of Aquatic Science and Technology (Eawag), Dübendorf, Switzerland.

Mara, D., J. Lane, B. Scott, and D. Trouba. 2010. Sanitation and health. PLoS Medicine 7(11): e1000363.

Marshall, M. N. 1996. Sampling for qualitative research. Family practice 13(6): 522-26. 
Marx, B., T. Stoker, and T. Suri. 2013. The economics of slums in the developing world. The Journal of Economic Perspectives: 187-210.

Mosler, H. J. 2012. A systematic approach to behavior change interventions for the water and sanitation sector in developing countries: a conceptual model, a review, and a guideline. International Journal of Environmental Health Research 22(5): 431-49.

Murray, A. and I. Ray. 2010. Commentary: Back-End Users: The Unrecognized Stakeholders in Demand-Driven Sanitation. Journal of Planning Education and Research 30(1): 94-102.

NISR. 2008. District Baseline Statistics: Gasabo and Nyarugenge Disticts. Kigali: National Institute of Statistics for Rwanda (NISR).

Okurut, R. Kulabako, J. M. Adogo, J. Chenoweth, S. Pedley, A. Tsinda, and K. Charles. 2013. Improved sanitation in low-income informal settlements - Acase of East African cities: Is it amyth? Water and Health Conference: Where Science meet Policy. UNC - Chapel Hill, USA.

Okurut, R. N. Kulabako, J. Chenoweth, and K. Charles. 2014a. Assessing demand for improved sustainable sanitation in low-income informal settlements of urban areas: a critical review. International Journal of Environmental Health Research(ahead-of-print): 1-15.

Outlaw, T., M. Jenkins, and B. Scott. 2007. Opportunities for Sanitation Marketing in Uganda. USAID Hygiene Improvement Project. pp. 62. Washington, DC: USAID.

Parry-Jones, S. 1999. Optimising the selection of demand assessment techniques for water supply and sanitation projects. Project/Task No: 207. pp. 46. London, UK: Water and Environmental Health.

Peal, A., B. Evans, and C. van der Voorden. 2010. Hygiene and Sanitation Software: An Overview of Approaches. pp. 157. Geneva, Switzerland: Water Supply \& Sanitation Collaborative Council.

Robbins, P. T. 2007. The reflexive engineer: perceptions of integrated development. Journal of International Development 19(1): 99-110.

Roma, E., C. Buckley, B. Jefferson, and P. Jeffrey. 2010. Assessing users' experience of shared sanitation facilities: A case study of community ablution blocks in Durban, South Africa. Water SA 36(5): 589-94.

Santos, A. C., J. A. Roberts, M. L. Barreto, and S. Cairncross. 2011a. Demand for sanitation in Salvador, Brazil: A hybrid choice approach. Social Science \& Medicine 72(8): 1325-32.

Santos, A. C., J. A. Roberts, M. L. Barreto, and S. Cairncross. 2011b. Demand for sanitation in Salvador, Brazil: A hybrid choice approach. Social Science \& Medicine.

Schertenleib, R. 2001. Principles and Implications of Household Centered Approach in Environmental Sanitation. First International Conference on Ecological Sanitation. pp. 2834. Duebendorf, Switzerland.

Sigel, K., K. Altantuul, and D. Basandorj. 2012. Household needs and demand for improved water supply and sanitation in peri-urban ger areas: the case of Darkhan, Mongolia. Environmental Earth Sciences 65(5): 1561-66.

Solo, T. M., E. Perez, and S. Joyce. 1993. Constraints in providing water and sanitation services to the urban poor. Washington, D.C.: US Agency for International Development.

Tumwebaze, C. G. Orach, C. Niwagaba, C. Luthi, and H.-J. Mosler. 2013. Sanitation facilities in Kampala slums, Uganda: users' satisfaction and determinant factors. International Journal of Environmental Health Research 23(3): 191-204.

Tumwebaze, I. K., C. B. Niwagaba, I. Günther, and H.-J. Mosler. 2014. Determinants of households' cleaning intention for shared toilets: Case of 50 slums in Kampala, Uganda. Habitat International 41: 108-13.

UBOS. 2011. 2011 Statistical Abstract. Uganda Bureau Of Statistics (UBOS), pp. 287. Kampala.

UN-HABITAT. 2003. The challenge of slums: global report on human settlements. Nairobi: United Nations Human Settlements Programme.

UN. 2002. Plan of Implementation of the World Summit on Sustainable Development. United Nations.

Van Der Geest, S. 2002. The night-soil collector: Bucket latrines in Ghana. Postcolonial Studies: Culture, Politics, Economy 5(2): 197-206. 
Van der Hoek, W., B. Evans, J. Bjerre, M. Calopietro, and F. Konradsen. 2010. Measuring progress in sanitation. In Reaching the MDG Target for Sanitation in Africa - A Call for Realism., pp. 51. Copenhagen: Ministry of Foreign Affairs of Denmark.

Van Wyk, R., D. Cousins, and A. Lagardien. 2004. Emerging themes from four case studies of sanitation delivery to informal settlements. In Water Institute of Southern Africa (WISA) Biennial Conference. Water Institute of Southern Africa (WISA).

Varley, R. C., M. M. Yacoob, and S. Smith. 1996. Beyond participation: locally based demand for environmental health in peri-urban areas. Washington, D.C.: Environmental Health Project.

Whittington, D., D. T. Lauria, A. M. Wright, K. Choe, J. A. Hughes, and V. Swarna. 1993. Household Demand for Improved Sanitation Services in Kumasi, Ghana: A Contingent Valuation Study. Water Reources Research 29(6): 1539-60.

WHO/UNICEF. 2010. JMP for Water Supply and Sanitation: Estimates for Improved Sanitation Facilities, Uganda 2010. pp. 15. Geneva: WHO / UNICEF.

WSP. 2001. Identifying Demand Drivers for Sanitation Technologies. The case of Ecosan in Africa. WSP, pp. 4. Nairobi: Water and Sanitation Program-Africa.

WSP. 2004. Sanitation and Hygiene in Kenya: Lessons on What Drives Demand for Improved Sanitation, pp. 12. Nairobi: Water and Sanitation Program-Africa (WSP). 